INPLASY

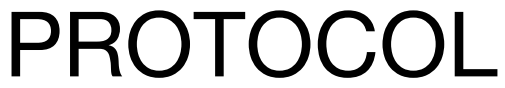

To cite: Deng et al. The Effect of Lidocaine on the prevention of Acute and Chronic Pain after Breast Cancer Surgery: A Meta-Analysis. Inplasy protocol 202130043. doi: 10.37766/inplasy2021.3.0043

Received: 14 March 2021

Published: 14 March 2021

Corresponding author:

Fang Deng

877589567@qq.com

Author Affiliation:

Department of Pain

Management of the Third

Xiangya Hospital of Central

South University

Support: None.

Review Stage at time of this submission: Preliminary searches.

Conflicts of interest: None declared.

\section{The Effect of Lidocaine on the prevention of Acute and Chronic Pain after Breast Cancer Surgery: A Meta- Analysis}

Deng, F1; Huang, D2; Liu, DB3; Zhou, HC4; Yu, SZ5.

Review question / Objective: Post-mastectomy pain syndrome (PMPS) is a significant complication of the treatment of breast cancer, with a prevalence of $2 \%$ to $78 \%$.It is a neuropathic pain condition localized in and around the area of surgery and lasting more than 3 months after surgery. PMPS can develop shortly or up to several months after surgery and can persist for many years. PMPS has a considerable negative influence on the quality of life of the affected women. Like other neuropathic pain conditions, the treatment is a difficult task. The amount of research on the perioperative prevention of PMPS is very limited and no consensus of the prevention of PMPS has yet been made. Therefore, it is of high clinical relevance to find a safe, reliable, and tolerated prevention with a substantial effect on PMPS.

INPLASY registration number: This protocol was registered with the International Platform of Registered Systematic Review and Meta-Analysis Protocols (INPLASY) on 14 March 2021 and was last updated on 14 March 2021 (registration number INPLASY202130043).

\section{INTRODUCTION}

Review question / Objective: Postmastectomy pain syndrome (PMPS) is a significant complication of the treatment of breast cancer, with a prevalence of $2 \%$ to $78 \%$.It is a neuropathic pain condition localized in and around the area of surgery and lasting more than 3 months after surgery. PMPS can develop shortly or up to several months after surgery and can persist for many years. PMPS has a considerable negative influence on the quality of life of the affected women. Like 
other neuropathic pain conditions, the treatment is a difficult task. The amount of research on the perioperative prevention of PMPS is very limited and no consensus of the prevention of PMPS has yet been made. Therefore, it is of high clinical relevance to find a safe, reliable, and tolerated prevention with a substantial effect on PMPS.

Condition being studied: Systemic administration of lidocaine has been shown to be superior to placebo to relieve neuropathic pain. Among patients undergoing abdominal surgery, intravenous lidocaine infusion in the perioperative period appears to lower pain scores, reduce postoperative analgesic requirements, facilitate return of bowel function, and decrease length of hospital stay. Nonetheless, its use in other surgical procedures remains controversial, and there is no conclusive evidence for clinically relevant benefits in the ambulatory setting. One systematic review (Chang et al., 2017) looked at the effects of intravenous lidocaine. Based on data from four studies, they reported a reduction of chronic pain at three to six months postoperatively. Their analysis on the outcome chronic pain was based only on two studies.It impossible to draw definite conclusions based on a limited number of small trials. More studies are warranted to clearly define the clinical impact of lidocaine infusion on chronic postmastectomy pain.

\section{METHODS}

Participant or population: Inclusion: Adults with breast cancer and undergoing breast surgery ;Exclusion: Adults with chronic neuropathic pain in the breast or chest region that is not post surgical or not post breast cancer related surgery.

Intervention: Lidocaine for breast cancer patients during perioperative period.

Comparator: Placebo or any other medications in breast cancer patients undergoing breast surgery.
Study designs to be included: We will include randomized controlled trials.

Eligibility criteria: Inclusion:Articles: (i) In English or chinese; (ii) Studies comparing effects of intravenous lidocaine with placebo or any other medications in patients undergoing breast surgery; (iii) with study design: randomized, controlled trials Exclusion: Articles: Conference abstracts, letters, announcements, registration information, unpublished studies

Information sources: 1.We searched the following databases:PubMed, Embase, web of science, The Cochrane Library (Cochrane Database of Systematic Reviews, Cochrane Central Register of Controlled Trials (CENTRAL)), CNKI, Wanfang Data, VIP Chinese Journal. 2. Citations identified through reference searching 3 Search dates: all literature dates included. 4.The searches will be rerun prior to the final analysis. 5.Restrictions: non-English language or non-Chinese language, animal studies.

Main outcome(s): The incidence of postmastectomy pain syndrome.

Additional outcome(s): The efficacy of the interventions on acute pain intensity; the cumulative opioid consumption the cumulative incidence of postoperative nausea and vomiting (PONV) during the first 24h.

Quality assessment / Risk of bias analysis: The quality of the included RCTs was assessed by two reviewers (AL, BR) independently, using the Cochrane Risk of Bias tool (Cochrane Handbook, version 5.1.0) including random sequence generation, allocation concealment, blinding of participant and personnel, blinding of outcome assessment, incomplete outcome data, selective reporting and other bias. Each item was rated as "low", "high" or "unclear" risk of bias. Discrepancies were discussed until an agreement was reached. 
Strategy of data synthesis: Conventional random effects meta-analyses (RevMan version 5.3, Cochrane Collaboration) were performed when at least two studies reported on the effect of an intervention on an outcome. When the I 2 of the research

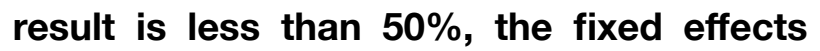
model is used, and when the I $2 \geq 50 \%$ of the research result, the random effects model is used for analysis. Among them, the relative risk (RR) and its $95 \%$ confidence interval $(\mathrm{Cl})$ are calculated from the count data, and the standardized mean difference (SMD) and its 95\% confidence interval $(\mathrm{Cl})$ are used for the measurement data.

Subgroup analysis: If numbers permit, subgroup analyses will be performed to assess the effect of the Lidocaine's dosage form, administration method, drug concentration, administration time and the effect of different surgical methods of consciousness on the outcome.

Sensitivity analysis: Sensitivity analysis will be used to test reliability and stability of the systematic review results, and to assess the source of heterogeneity. We will compare the results before and after by excluding trials with a high risk of bias or eliminating trials with a high risk of bias or eliminating each study individually one study each time and then pooling the remaining studies.

Country(ies) involved: China.

Keywords: Lidocaine, intravenous infusion, breast cancer surgery, chronic pain, mate analysis, acute pain.

Contributions of each author:

Author 1 - Fang Deng.

Email: 877589567@qq.com

Author 2 - Dong Huang.

Email: huangdong@csu.edu.cn

Author 3 - DongBiao Liu.

Email: 1309893927@qq.com

Author 4 - HaoCheng Zhou.

Author 5 - Sanzi Yu. 